\title{
Correction to: Plasma concentrations of remdesivir metabolite in a critical COVID-19 patient needing continuous venovenous haemodialysis
}

\author{
Massimo Tempestilli ${ }^{1}$. Giulia Valeria Stazi ${ }^{1}$ - Gaetano Maffongelli ${ }^{1}$ Maria Cristina Marini ${ }^{1}$. \\ Tommaso Ascoli Bartoli ${ }^{1}$. Giuseppe Ippolito ${ }^{1}$. Emanuele Nicastri ${ }^{1}$ - Luisa Marchioni ${ }^{1}$. Chiara Agrati ${ }^{1}$ (D)
}

Published online: 3 March 2022

(c) Springer-Verlag GmbH Germany, part of Springer Nature 2022

\section{Correction to: European Journal of Clinical Pharmacology (2021) 77:1583-1585 https://doi.org/10.1007/s00228-021-03128-7}

In the Acknowledgments section there is a typing error in the code number of the project is COVID-2020-12371675.

Publisher's Note Springer Nature remains neutral with regard to jurisdictional claims in published maps and institutional affiliations.

The original article can be found online at https://doi.org/10.1007/ s00228-021-03128-7.

\footnotetext{
Chiara Agrati

chiara.agrati@inmi.it

Immunology and Pharmacology Laboratory, National Institute for Infectious Diseases "Lazzaro Spallanzani" IRCCS, via Portuense, 292, 00149 Rome, Italy
} 